\title{
CHEMILUMINESCENCE USED IN BIOCHEMICAL INVESTIGATIONS. AN APPLICATION OF THE LANTHANIDE IONS
} AS A CHEMILUMINESCENT PROBE

\author{
M. Elbanowski, K. Staniński and M. Kaczmarek \\ Department of Rare Earths, Faculty of Chemistry, Adam Mickiewicz University \\ Grunwaldzka 6, 60-780 Poznan, Poland
}

This article concerns problems related to chemiluminescence and briefly fluorescence, which will be discussed. It also gives a review of application of the chemiluminescent method in chemistry, biology and medicine, in the absence of lanthanide ions and in their presence.

PACS numbers: 78.60.Ps

In this review the following important problems are covered:

1. Chemiluminescence (CL) as a phenomenon and a method of study.

2. The occurrence of CL in chemistry, biology and medicine (without the presence of lanthanide ions, $\operatorname{Ln}(\mathrm{III}))$.

3. Importance of the lanthanide ions in the investigations discussed.

4. The role of the fluorescent method in the studies.

5. Our own studies on the applications of the CL method with $\operatorname{Ln}$ (III) ion as a spectroscopic probe.

\section{Chemiluminescence as a phenomenon and a method of study}

Chemiluminescence [1-3], similarly as bioluminescence, is still a very interesting phenomenon and a modern spectroscopic method used mainly in chemistry, biology and medicine. The discussed method may be applied in investigation of many problems in these fields.

Chemiluminescence is an ultra-weak emission of light generated as a result of chemical reaction. As CL is a complicated process, it can be, in general, divided into two steps: the first - the excitation stage connected with generation of excited molecule identical with the excited product, and the second - the luminescence stage at which the excited molecule is deactivated to its ground state emitting a quantum of radiation. The first stage is of chemical nature, while the second - 
of physical and combines several processes. In this problem the energy transfer and quenching processes of CL play an important role especially interesting in biological molecules.

The efficiency of light emission is expressed as a quantum yield. The overall quantum yield of a chemiluminescent reaction $\left(\Phi_{\mathrm{CL}}\right)$ is the main parameter which characterizes transformation of chemical energy into radiation, and is defined as the number of photons emitted per number of molecules involved in the reaction. The following equation presents the components of $\Phi_{\mathrm{CL}}$ :

$$
\Phi_{\mathrm{CL}}=\Phi_{\mathrm{C}} \Phi_{\mathrm{Ex}} \Phi_{\mathrm{F}}
$$

where $\Phi_{\mathrm{C}}-$ means a chemical yield; fractions of molecules going through the main reaction (excluding "dark" side reaction); $\Phi_{\mathbf{E x}}$ - fraction of those molecules which react in the chemiluminescence pathway and become electronically excited; $\Phi_{F}-$ fluorescence quantum yield of the excited product, i.e. the number of photons emitted, divided by the number of molecules actually excited. These parameters $\left(\Phi_{\mathrm{C}}, \Phi_{\mathrm{Ex}}, \Phi_{\mathrm{F}}\right)$ making up $\Phi_{\mathrm{CL}}$ can be estimated independently but with a different level of difficulty.

The ultra-weak chemiluminescent reactions often reveal extremely low quantum yields $\left(\Phi_{\mathrm{CL}}=10^{-3} \div 10^{-8}\right.$, but also $\left.10^{-9} \div 10^{-15}\right)$.

In general, the efficient CL of chemical compounds is a result of oxidation involving molecular oxygen or hydrogen peroxide.

\section{The occurrence of chemiluminescence in chemistry, biology and medicine (without the presence of lanthanide ions)}

There are many well-known examples of occurrence of chemiluminescence phenomenon, as well as application of that method in solving different problems of chemistry, biology and medicine and when the lanthanide ions are not used as luminescent probes. Below there are examples of phenomena in which CL has been observed or in investigation of which it has been used as a method [1, 2]:

- the upper layers of atmosphere (90-350 km) where an "airglow" and "air afterglow" appear; in that situation ultraviolet radiation from the sun causes dissociation and ionization of atoms and molecules. Recombination of these reactive species results in light emission. Therefore many spectral lines can be observed: e.g. atomic $\mathrm{O}, \mathrm{Na}, \mathrm{H}, \mathrm{Ca}$ and $\mathrm{K}$;

- the flames containing e.g. $\mathrm{N}_{2} \mathrm{O}, \mathrm{H}_{2} \mathrm{~S}, \mathrm{CO}, \mathrm{CS}_{2}$ and $\mathrm{CN}$ in air or oxygen;

- gas phase reactions involving metal vapours;

- the synthesized large group of organic compounds, where the oxidation reactions or electron transfer and radical-type reactions take place (for example the lophine, triphenylimidazole, first synthesized by B. Radziszewski (Lvov, 1877) and other important compounds of this group: luminol, 5-aminophthalhydrazide and lucigenin, bis- $N$-methyl acridinium nitrate);

- activated phagocytes (such as neutrophils) enhanced by added luminol and lucigenin (as synthetic compounds); thus obtained ultra-weak CL serves as an indicator of reactive oxygen metabolites, peroxidase, oxidase, oxygenase process, and lipid peroxidation in cells; 
- energy transfer processes (inter/intra-molecular) occurring in many luminous organisms, and in the case of the synthetic compounds, e.g. oxidation reactions (with $\mathrm{H}_{2} \mathrm{O}_{2}$ or other oxygen containing oxidants), reactions of peroxyesters, decomposition of dioxetans or dioxetanones, reactions producing singlet oxygen;

- electron transfer reactions (chemically initiated electron exchange luminescence - CIEEL); in 1961 Linschitz had explained CL of tetralin peroxide catalysed by porphyrin, i.e. charge transfer complex forms allowing electron exchange between the product of the chemical reaction, the donor, and the fluorescent acceptor, leading to excitation of the fluorophore which then emits radiation; decomposition reaction of a diacyl peroxide, diphenoyl peroxide, to benzocoumarin and $\mathrm{CO}_{2}$, when the electron exchange mechanism takes place; in the chemiluminescent reactions of some types of flies (e.g. fireflies), an important role is played by the intramolecular electron transfer processes;

- analytical applications - with enormously high sensitivity and detection of traces of substances - in the range even to $10^{-21} \mathrm{~mol}$ - for many synthetic and natural compounds useful in a solution of many different biochemical processes and biological cells; for example using the alkaline solutions of strongly luminous luminol and hydrogen peroxide, $10^{-6} \%$ of haemin can be determined; the traces of $\mathrm{SO}_{2}$ in pure water $(0.8-100 \mathrm{ng} \mathrm{SO} 2 / \mathrm{ml})$ are also determined, as well as $6 \times 10^{-9} \mathrm{~mol} / \mathrm{l}$ $\mathrm{H}_{2} \mathrm{O}_{2}(0.2 \mu \mathrm{g} / \mathrm{l})$ by CL measurement in the system: peroxalate-perylene- $\mathrm{H}_{2} \mathrm{O}_{2}$;

- biomedical investigations - as a method allowing a solution of different biological problems and make a medical decision; these problems are, among others, connected with: the measurement of reactive oxygen metabolites produced by cells, estimation of enzymes and metabolites in tissue with participation firefly luciferin-luciferase, measurement of intracellular free $\mathrm{Ca}$ (II);

- microbiological and biotechnological problems concerning detection of small numbers of microorganisms, such as bacteria, viruses and other species.

\section{Importance of the lanthanide ions in the investigations discussed}

Some of the lanthanide ions, mainly $\mathrm{Eu}(\mathrm{II}), \mathrm{Eu}(\mathrm{III})$ and $\mathrm{Tb}(\mathrm{III})$, showing strong luminescence, can be used as luminescent probes. [4, 5], i.e. as chemiluminescent and fluorescent probes, activators and energy transformers (donors or acceptors) in biological systems.

The europium and terbium ions can be isomorphously exchanged with the main $\mathrm{Na}, \mathrm{K}, \mathrm{Mg}$ and $\mathrm{Ca}$ cations present in the natural biological systems, where they participate in different catalytic and transport processes, as well as initiators of chain-reactions. The europium and terbium ions can be used, as the luminescent probes, in determination of the binding sites of metal ions with biochemical molecules and the structure, dynamics and activity of the biological systems.

We are particularly interested in using the $\mathrm{Eu}(\mathrm{II}) / \mathrm{Eu}$ (III) ion system, which can function as a chemiluminescent probe after its insertion into the biochemical and macrocycle compounds.

The application of the lanthanide ions as the chemiluminescent probes till now has not been used as frequent as fluorescent probes and it is still an open 
field for investigation because of low intensity of CL observed particularly in studies of biochemical systems. Hence, when the energy transfer from biomolecule to the lanthanide ion is involved and when such compounds, as eosine, showing high quantum efficiency of luminescence are applied, the intensity of CL can be considerably increased.

In studies of systems containing biochemical compounds with lanthanide ions as probe, the complexation reactions play a very important role. Therefore, the complexes of the lanthanide ions of high luminescence quantum yield with the biochemical compounds can considerably increase the CL intensity as a result of energy transfer from the investigated biomolecule to the lanthanide ion. In this case, the $\mathrm{Eu}$ (III)-chelate complexes can increase the CL intensity by $10^{3}-10^{4}$ times in the reaction of hydrocarbons oxidation.

As follows from a comparison between the CL spectra originated as a result of the oxidation reactions of the lipids in liposomes, when the lanthanides do not participate in these systems, and with the participation of the $\mathrm{Tb}(\mathrm{III})$ ions and the Eu(III) complex with tetracycline, the light sum $\left(\sum I \Delta t\right)$ of observed CL can be considerably increased [6].

It is also worth mentioning that other problems, where lanthanides take part in the chemiluminescent reactions, have been also studied. These are the examples:

- the reduction reaction of $\mathrm{Tb}(\mathrm{IV})$ and $\operatorname{Pr}(\mathrm{IV})$ in aqueous solutions of polywolframate [7];

- the dissolving reaction of double lithium and terbium(IV) oxide in acids [8];

- hydrolysis of the cyclopentadienyl compounds of $\mathrm{Eu}$ (II and III) and Sm(II and III) [9];

- chemiluminescent system containing Eu(III)-1, 2-dioxetan [10];

- chemiluminescent reaction of samarium with $\mathrm{SF}_{6}$ and $\mathrm{CCl}_{4}$ in gas phase [11].

\section{The role of the fluorescent method in the studies}

The fluorescence method is also used in the study of systems containing the lanthanide ion (mainly $\mathrm{Eu}(\mathrm{III})$ and $\mathrm{Tb}(\mathrm{III})$ ) and the molecules of biological interest [12]. These systems can serve, for example:

- in the investigation of changes in the biomolecules structure and the active centres present in the enzymes;

- in the differentiation of the structure features of DNA on the base of the emission spectra of $\mathrm{Tb}(\mathrm{III})$;

- in the study of energy transfer processes.

\section{Our own studies on the applications of the CL method with $\operatorname{Ln}(\mathrm{III})$ ion as a spectroscopic probe}

The results of our investigations already partly published, or the new ones will be now presented.

The system consisting of biochemical compounds, $\mathrm{Eu}(\mathrm{II}) / \mathrm{Eu}(\mathrm{III})$ ions and oxidant $\left(\mathrm{H}_{2} \mathrm{O}_{2}\right)$ is the basis of our investigations. The emissive $\mathrm{Eu}$ (II)/ $\mathrm{Eu}$ (III) ions, as a chemiluminescent probe, after complexation of the $\mathrm{Eu}(\mathrm{II})$ ions by the 
biomolecule and after oxidizing them to $\mathrm{Eu}$ (III) by $\mathrm{H}_{2} \mathrm{O}_{2}$, permit observation of the enhanced intensity of CL, originating from the excited $\mathrm{Eu}(\mathrm{III}){ }^{5} D_{0}$ state. The obtained variable values of CL intensity (at $\lambda_{\mathrm{Eu}(\mathrm{III})}=590 \div 620 \mathrm{~nm}$ ) indeed prove directly the $\mathrm{Eu}(\mathrm{III})$ emission, but can be a consequence of the characteristic features of biomolecules, such as a complex composition and configuration and hence different interactions, as well as their conformational changes.

Discussion of the obtained results must also take into account the influence of $\mathrm{H}_{2} \mathrm{O}_{2}$ and its radicals $\left(\mathrm{OH}, \mathrm{O}_{2}^{-}\right)$on the studied biomolecules, and hence a possibility of creation of different types of the hydroxy- and peroxy-derivatives of these molecules.

A simplified scheme of the apparatus for CL measurements in our investigations is shown in Fig. 1.

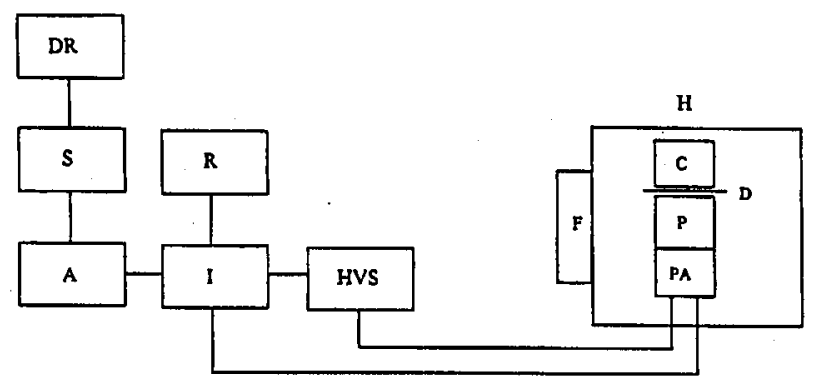

Fig. 1. Scheme of the apparatus for chemiluminescence measurements. DR - digital recorder ERD-103; S - scaler P-21; A - analyzer A-22M; R - recorder VAREG; I integrator ILL-21a; HVS - high voltage supplier; PA - preamplifier; P - photomultiplier; $\mathrm{C}-$ measurement cell; $\mathrm{F}$ - filters; $\mathrm{D}$ - diaphragm; $\mathrm{H}$ - housing.

Chemiluminescence was measured by the single-photon counting method using a photomultiplier. The spectral distribution of CL was estimated with a set of calibrated cut-off filters covering the spectral region about 400-700 nm.

Changes of the integral CL intensity $\left(\sum I \Delta t\right)$ have been recorded as a function of the optimum experimental conditions, mainly $\mathrm{pH}$, the concentration of europium ions and the molar ratios of the system components (Eu(II)/Eu(III): biochemical complexing agent). For better appreciation of the phenomena, the overall quantum yield of the ultra-weak CL have been estimated using the standard reaction.

In the fluorescent study of the Eu(III)-ATP system the influence of $\mathrm{pH}$ and the molar ratios of the components on the fluorescence intensity has been analysed. The corrected fluorescence spectra of the solutions were recorded with a Perkin-Elmer MPF-3 and MPF-44 spectrofluorimeters.

The adenosine nucleotides - ATP, ADP, AMP and c-AMP, playing a very important role among biochemical, high-energy molecules, have been investigated [13]. The highest observed CL intensity (its maximum, $I_{\max }$, as a peak height), as well as the integral intensity (light sum, $\sum I \Delta t$ ) of CL, originated from the $\mathrm{Eu}(\mathrm{II}) / \mathrm{Eu}(\mathrm{III})$-nucleotide- $\mathrm{H}_{2} \mathrm{O}_{2}$ system, depends on the number of phosphates, 
i.e. the $\mathrm{P}-\mathrm{O}-$ groups, present in the nucleotide molecules. The observed dependence gives a sequence: ATP > ADP > c-AMP > AMP. Stability of particular lanthanide-nucleotide complexes is consistent with the CL intensity obtained for these systems. The values of their stability constants are in the range of $10^{7}$ for ATP-Ln, to $10^{2}$ for AMP-Ln. These results confirm a great significance of ATP as a leading member of the nucleotide group.

The observed CL shows very low values of quantum yield. They are of the order of $10^{-12}$ for uncomplexed europium(II) and europium complexed with AMP, and $10^{-10}$ for europium complexed with ATP under optimum conditions.

The spectral distribution of $\mathrm{Eu}$ (III) CL originated from the $\mathrm{Eu}(\mathrm{II}) / \mathrm{Eu}$ (III)ATP $-\mathrm{H}_{2} \mathrm{O}_{2}$ system indicates the occurrence of the following bands: $\lambda=590 \div 620$, and $c a .700 \mathrm{~nm}$ ascribed to CL of Eu(III) ions complexed by ATP, corresponding to the appropriate $\mathrm{Eu}(\mathrm{III})$ transitions: a magnetic dipole transition ${ }^{5} D_{0}-{ }^{7} F_{1}(\lambda=$ $594 \mathrm{~nm})$, a hypersensitive transition ${ }^{5} D_{0}-{ }^{7} F_{2}(\lambda \simeq 620 \mathrm{~nm})$ and a forced electric dipole transition ${ }^{5} D_{0}-{ }^{7} F_{4}(\lambda \simeq 705 \mathrm{~nm})$; observed $\lambda=470 \div 480 \mathrm{~nm}$ can be attributed to the $\mathrm{CO}_{3}^{2-}$ ions present in the solution.

The fluorescence spectra of $\mathrm{Eu}(\mathrm{III})$ ions and of $\mathrm{Eu}(\mathrm{III})-\mathrm{ATP}$ complexes obtained at different $\mathrm{pH}$ values ( 2 and 7 ) show that complexation of $\mathrm{Eu}(\mathrm{III})$ causes an increase in the fluorescence intensity, especially of the hypersensitive band, at $\lambda \simeq 620 \mathrm{~nm}$, due to a decrease in radiationless transitions in this system.

Luminescence spectroscopy of lanthanide ions complexed by ligands of biological importance has been successfully used for obtaining information on the energy levels of the excited biochemical molecules and the energy transfer processes, very important in the biological systems. In this respect, the ${ }^{5} D_{0}-{ }^{7} F_{2}$ hypersensitive transition of $\mathrm{Eu}(\mathrm{III})$ is especially suitable.

In the fluorescence investigation, taking into account the energy transfer process, the creation of the emission bands of the Eu(III) ions (at $\lambda \simeq 594$ and 620 $\mathrm{nm}$ ) has been found having used the excitation wavelength $\lambda_{\max }=320 \mathrm{~nm}$ (the region of ATP molecules absorption). The intramolecular energy transfer process takes place from the ATP molecule to the Eu(III) ion. This energy transfer is most likely a result of participation of the active $\mathrm{P}-\mathrm{O}-$ groups present in the nucleotide molecules. Similar investigations have been carried out with $\mathrm{Eu}$ (III)-ADP or c-AMP or AMP complexes, for which the energy transfer has been also observed but with lower intensity.

It seems very likely that a similar intramolecular energy transfer process can take place also in the $\mathrm{Eu}(\mathrm{II}) / \mathrm{Eu}(\mathrm{III})$-nucleotide $-\mathrm{H}_{2} \mathrm{O}_{2}$ system, and probably analogously in some natural, biological systems where the transformation processes can be discussed.

At low concentration of ATP (below $30 \mathrm{mM}$ ), the maximum of absorption band at $\lambda=260 \mathrm{~nm}$, and fluorescence band at $\lambda=360 \div 380 \mathrm{~nm}$ are observed [12]. In these conditions radiationless, intramolecular energy transfer can occur from the triplet state of ATP to the ${ }^{5} L_{6}$ level of Eu(III) at $\lambda=394 \mathrm{~nm}$, which is a fundamental stage for fluorescence of $\mathrm{Eu}$ (III) emitted from the ${ }^{5} D_{0}$ excited state observed in our study. That proper proposed energy level diagram showing an intramolecular energy transfer process from ATP to $\mathrm{Eu}(\mathrm{III})$ in the molecule of the Eu(III)-ATP complex is presented in Fig. 2. 


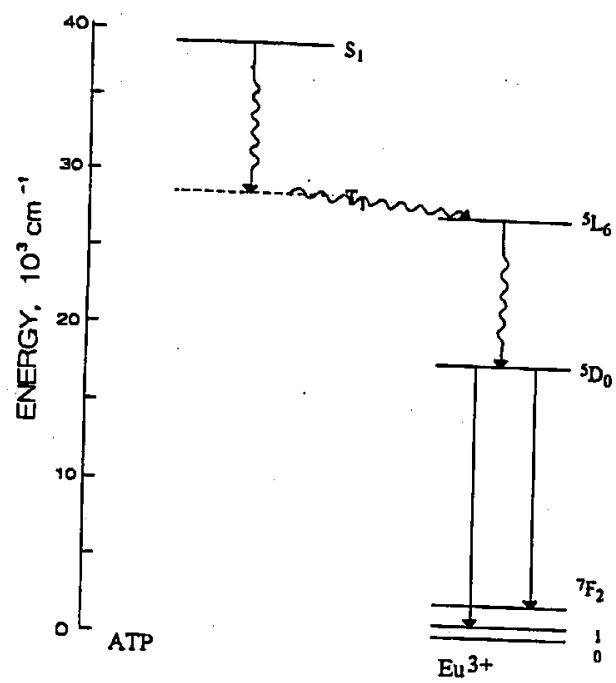

Fig. 2. Energy level diagram presenting the intramolecular energy transfer process from ATP to $\mathrm{Eu}(\mathrm{III})$ in the molecule of Eu(III)-ATP complex.

The reduced nicotinamide adenine nucleotide (NADH) molecule is another relevant biochemical compound, related to the nucleotides and extending this group [14]. As follows from a comparison of the reaction course, formation time and $\mathrm{CL}$ intensity of $\mathrm{Eu}(\mathrm{II}) / \mathrm{Eu}(\mathrm{III})-\mathrm{NADH}-\mathrm{H}_{2} \mathrm{O}_{2}$ system under investigation and the $\mathrm{Eu}(\mathrm{II}) / \mathrm{Eu}$ (III)-ATP- $\mathrm{H}_{2} \mathrm{O}_{2}$ system, CL intensity is much higher and its duration longer in the case of ATP than NADH. The system containing NADH emits immediately after insertion of $\mathrm{H}_{2} \mathrm{O}_{2}$ into a solution containing $\mathrm{Eu}(\mathrm{II}), \mathrm{NADH}$ and phosphate buffer of $\mathrm{pH}=7$. After $4 \mathrm{~s}$, the CL intensity reached the maximum and its duration was about $30 \mathrm{~s}$.

Chemiluminescence was generated by the processes taking place in these systems, particularly oxidation of $\mathrm{Eu}$ (II) to $\mathrm{Eu}$ (III) by $\mathrm{H}_{2} \mathrm{O}_{2}$ and the radical reactions resulting from it.

In order to give a more complete account of the investigated systems, the quantum yield of CL was determined. On the basis of the obtained $\Phi_{C L}$ results for a series of the nucleotides, one can claim that $\Phi_{\mathrm{CL}}$ decreases in the sequence $\operatorname{ATP}\left(3 \times 10^{-10}\right)>\operatorname{ADP}\left(1 \times 10^{-11}\right)>$ NADH $\left(5 \times 10^{-12}\right) \geq \mathrm{c-AMP}\left(3 \times 10^{-12}\right)$ together with a decrease in the number of $\mathrm{P}-\mathrm{O}-$ groups present in the particular nucleotide molecules, associated with their energy.

In order to perform an analysis of the observed CL for the NADH system, its spectral distributions was determined. The comparison of these results with those obtained for the system containing ATP or ADP and AMP, shows that in all these cases identical bands occur, which are ascribed to the same wavelengths. This allows one to draw a conclusion that similar processes take place in the systems containing these nucleotides, thus determining the similar character of the CL 
obtained. The observed emission originates from $\mathrm{Eu}$ (III) ions, which is manifested by the bands at $\lambda=590 \div 620 \mathrm{~nm}$.

The course of processes leading to CL observation, taking into account mainly the generation of $\mathrm{Eu}(\mathrm{III})$, may be described by the following reactions:

$$
\begin{aligned}
\mathrm{Eu}(\mathrm{II})+\mathrm{H}_{2} \mathrm{O}_{2} & \longrightarrow \mathrm{Eu}(\mathrm{III})+\mathrm{OH}^{-}+\mathrm{OH}^{-} \\
\mathrm{OH}+\mathrm{Eu}(\mathrm{II}) & \longrightarrow \mathrm{Eu}(\mathrm{III})+\mathrm{OH}^{-} \\
\mathrm{Eu}(\mathrm{III}) & \longrightarrow \mathrm{Eu}(\mathrm{III})^{*} \\
\mathrm{Eu}(\mathrm{III})^{*} & \longrightarrow \mathrm{Eu}(\mathrm{III})+h \nu_{\mathrm{CL}} .
\end{aligned}
$$

These processes may also lead to successive reactions in the studied system as a result of which excited molecules of singlet oxygen may be formed. The CL bands at $\lambda=460 \div 480 \mathrm{~nm}$ and $\lambda=625 \div 640 \mathrm{~nm}$ may be assigned to the radiative transitions of the excited ${ }^{1} \mathrm{O}_{2}$ which proceed according to the following equations:

$$
\begin{aligned}
& { }^{1} \mathrm{O}_{2}\left({ }^{1} \Delta_{\mathrm{g}}{ }^{1} \Sigma_{\mathrm{g}}^{+}\right) \longrightarrow 2^{1} \mathrm{O}_{2}\left({ }^{3} \Sigma_{\mathrm{g}}^{-}\right)+h \nu(476 \mathrm{~nm}) \\
& { }^{1} \mathrm{O}_{2}\left({ }^{1} \Delta_{\mathrm{g}}{ }^{1} \Delta_{\mathrm{g}}\right) \longrightarrow 2^{1} \mathrm{O}_{2}\left({ }^{3} \Sigma_{\mathrm{g}}^{-}\right)+h \nu(634 \mathrm{~nm}) .
\end{aligned}
$$

The strong band in the range $\lambda=460 \div 480 \mathrm{~nm}$ may be partly related to $\mathrm{CO}_{3}^{2-}$ groups present in the studied solutions.

Because of the presence of $\mathrm{H}_{2} \mathrm{O}_{2}$ in the system, it is necessary to take into account the radical mechanism in the process of generation of CL. Therefore a study of CL quenching was carried out. It follows from the performed tests that mannitol and $n$-butanol (for $\mathrm{OH}$ radicals) quenched CL only slightly (ca. 5\%), and $\beta$-naphthol induced a slightly larger quenching, whereas L-cysteine (for $\mathrm{HO}_{2}$ radicals) brought about a considerable decrease in the CL intensity (about $40 \%$ ). These results testify to a radical character of the studied reactions leading to the generation of CL.

Among the large molecules playing an important role from the biological point of view, deoxyribonucleic acid (DNA) molecules are noteworthy. This complex structure needs different methods of investigations giving a chance for elucidation some processes and mechanisms taking place in this system.

The system $\mathrm{Eu}(\mathrm{II}) / \mathrm{Eu}(\mathrm{III})-\mathrm{DNA}-\mathrm{H}_{2} \mathrm{O}_{2}$ has been studied [15] at two different values of $\mathrm{pH}$, i.e. 6 and 9. The addition of $\mathrm{Eu}(\mathrm{II})$ ions to the $\mathrm{DNA}-\mathrm{H}_{2} \mathrm{O}_{2}$ system caused a strong increase in CL intensity. The observed shapes of the CL curves imply that mainly an alkaline environment is responsible for intensive CL.

A spectral distribution of CL obtained from the $\mathrm{Eu}(\mathrm{II}) / \mathrm{Eu}(\mathrm{III})-\mathrm{DNA}-\mathrm{H}_{2} \mathrm{O}_{2}$ system at $\mathrm{pH}=6$ has shown that the strongest bands at the wavelengths $\lambda=$ $586 \div 600 \mathrm{~nm}$ resulted from the $\mathrm{Eu}(\mathrm{III})$ emission and the others rather weak ones, at $\lambda=460 \div 480$ and $665 \div 680 \mathrm{~nm}$, are connected with the creation of the singlet oxygen ${ }^{1} \mathrm{O}_{2}$. The first band may be also partly related to the $\mathrm{CO}_{3}^{2-}$ groups present in the studied solutions.

The mechanism of $\mathrm{CL}$ generated from the system containing DNA at $\mathrm{pH}=6$, using the radical quenchers, has also been investigated. The following quenchers of $\mathrm{CL}$ have been used: for $\mathrm{OH}^{\prime}$ radicals $-n$-butanol and mannitol; for $\mathrm{HO}_{2}^{-}$radicals - L-cysteine; for $\mathrm{O}_{2}^{-\cdot}$ radicals - tiron; for ${ }^{1} \mathrm{O}_{2}-\beta$-carotene. The results of quenching suggest a radical character of the investigated reactions leading to the generation of CL. 
A spectral distribution of $\mathrm{CL}$ in the $\mathrm{Eu}(\mathrm{II}) / \mathrm{Eu}(\mathrm{III})-\mathrm{DNA}-\mathrm{H}_{2} \mathrm{O}_{2}$ at $\mathrm{pH}=9$ is different than for the same system but at $\mathrm{pH}=6$. At $\mathrm{pH}=9$ the main band at $\lambda=460 \div 480 \mathrm{~nm}$ is shown, instead the characteristic band of $\mathrm{Eu}$ (III) ions is, in fact, not observed. There is a lack of the Eu-DNA complex in the system at $\mathrm{pH}=9$. Two separate main processes may appear here: interaction of $\mathrm{H}_{2} \mathrm{O}_{2}$ with DNA molecules and the oxidation of $\mathrm{Eu}(\mathrm{II})$ to $\mathrm{Eu}(\mathrm{III})$ ions by $\mathrm{H}_{2} \mathrm{O}_{2}$. This main chemiluminescent band (at $\lambda=460 \div 480 \mathrm{~nm}$ ) at $\mathrm{pH}=9$ probably results from the first process.

At present we are interested in application of the CL method while using the lanthanide ions for the medical purposes. The first main aim of the work is the characterization, by means of this method, of the plasma samples originated from the patients with chronic renal failure put to the hemodialysis (before and after that procedure). The system studied at $\mathrm{pH}=7$ consists of plasma sample $\mathrm{Eu}(\mathrm{II}) / \mathrm{Eu}(\mathrm{III})$ and $\mathrm{H}_{2} \mathrm{O}_{2}$ which is an oxidizing agent for oxidation of $\mathrm{Eu}(\mathrm{II})$, complexed by plasma's components, to $\mathrm{Eu}(\mathrm{III})$.

First, we have obtained definite kinetic curves of CL, as well as the characteristic spectral distribution for the plasma of chosen patients [16]. These results have shown the differences between particular samples of patient's plasmas, as well as between those samples and the samples taken from healthy people. But these investigations did not give all the information needed. The complex and very rich structure of the plasma was a chief obstacle. A great variety of natural components of plasma has not permitted so far giving a complete explanation of the observed changes in chemiluminescent spectra.

Now, we continue this direction of work but simultaneously our interest is more concentrated on the study of the plasma's water and the dialysate (i.e. the dialysing physiological liquid, containing mainly salts). Both systems do not contain a great variety of components, particularly the high-molecular ones.

The plasma's water, obtained by means of the dry ultrafiltration process, similarly as the dialysate, have been studied, using the chemiluminescent method, in the $\mathrm{Eu}(\mathrm{II}) / \mathrm{Eu}$ (III)-plasma's water or dialysate- $\mathrm{H}_{2} \mathrm{O}_{2}$ systems. On the basis of the first obtained results we can find that the courses of the kinetic curves of CL for the dialysate samples, obtained before and after the contact with circulating blood of the particular patients, are partly different. The important role can be played by different natural substances partly removed from the blood in the course of hemodialysis. The analysis of the initial results of the plasma's water systems shows an influence of creatinine (a natural component of this plasma's water) on the courses of the observed CL.

But till now we do not know about the influence of other natural components of the studied systems on the character of CL spectra. Those systems are currently being studied.

\section{References}

[1] A.K. Campbell, Chemiluminescence: Principles and Applications in Biology and Medicine, Ellis Horwood Ltd., Chichester and VCH Verlagsgesellschaft mbH, Weinheim 1988 . 
[2] K.van Dyke, Bioluminescence and Chemiluminescence: Instruments and Applications, Vols. 1 and 2, CRC Press Inc., Boca Raton (Florida) 1985.

[3] R.F. Vasilev, Usp. Khim. 39, 1130 (1970).

[4] M. Albin, W.DeW. Horrocks, Jr., Inorg. Chem. 24, 895 (1985).

[5] F.S. Richardson, Chem. Rev. 82, 541 (1982).

[6] V.S. Sharov, Yu.A. Vladimirov, Biofizika 27, 327 (1982).

[7] A.B. Yusov, A.M. Fedoseev, W.I. Spitsin, Dokl. Akad. Nauk SSSR 289, 310 (1986).

[8] A.B. Yusov, A.M. Fedoseev, Radiokhimia 5, 16 (1989).

[9] R.G. Bulgakov, W.P. Kazakov, Dokl. Akad. Nauk SSSR 304, 114 (1989).

[10] G.L. Sharipov, A.I. Voloshin, W.P. Kazakov, G.A. Tolstikov, Dokl. Akad. Nauk SSSR 315, 425 (1990).

[11] U.B. Pavanaja, P.D. Naik; A.V. Sapre, P.N. Bajaj, K.V.S. Rama Rao, J.P. Mittal, R.M. Iyer, J. Photochem. 38, 43 (1987).

[12] R. Reisfeld, S. Nathanson, E. Greenberg, J. Phys. Chem. 80, 2538 (1976).

[13] M. Elbanowski, M. Paetz, J. Sławiński, L. Cieśla, Photochem. Photobiol. 47, 463 (1988).

[14] M. Elbanowski, M. Paetz, J. Sławiński, E. Ludwiczak, J. Photochem. Photobiol. A: Chem. 62, 27 (1991).

[15] M. Paetz, M. Elbanowski, J. Photochem. Photobiol. A: Chem. 55, 63 (1990).

[16] M. Elbanowski, H. Wysocki, M. Paetz, R. Czarnecki, E. Ludwiczak, A. Minczykowski, in: Abstracts of IIIrd Internat. Symp. on Quantitative Luminescence Spectrometry in Biomedical Sciences, State University of Ghent, Ghent 1989, p. 117. 